\title{
ANNALS OF

\section{Precision Medicine Based on High-Throughput Nucleotide Sequencing for a Patient with SCN2A-Related Developmental Epileptic Encephalopathy}

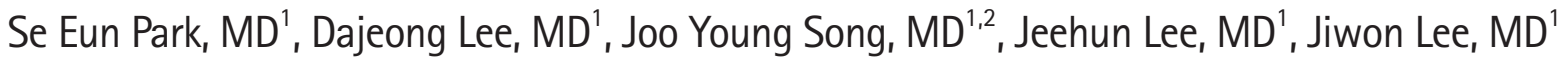 \\ ${ }^{1}$ Department of Pediatrics, Samsung Medical Center, Sungkyunkwan University School of Medicine, Seoul, Korea \\ ${ }^{2}$ Department of Pediatrics, CHA Gangnam Medical Center, CHA University, Seoul, Korea
}

Received: September 16, 2020

Revised: October 5, 2020

Accepted: October 5, 2020

Corresponding author:

Jiwon Lee, MD

Department of Pediatrics, Samsung

Medical Center, Sungkyunkwan

University School of Medicine, 81

Irwon-ro, Gangnam-gu, Seoul

06351, Korea

Tel: +82-2-3410-0811

Fax: +82-2-3410-0043

E-mail:jwjw.lee@samsung.com
The voltage-gated sodium channel, type II, alpha (SCN2A) gene (OMIM 182390) encodes the alpha subunit of $\mathrm{Na}_{\mathrm{v}} 1.2$ [1]. It is expressed plentifully in neurons during early infancy and plays a role in neuronal excitability [2]. SCN2A variants are one of the causes of neurodevelopmental disorders, accounting for $1 \%$ of all epileptic encephalopathies [3].

There are several epilepsy phenotypes associated with $S C N 2 A$ variants: benign familial neonatal-infantile seizures, neonatal seizures and late onset episodic ataxia, developmental epileptic encephalopathy, and autism spectrum disorders. In addition, there are many seizure types associated with SCN2A variants, including focal clonic seizures, focal tonic seizures, generalized tonic clonic seizures, hemi-clonic seizures, spasms, myoclonic seizures, and atonic seizures [4]. The phenotypic variability associated with $S C N 2 A$ variants has been reported in the review of literatures $[3,4]$. The location of the variants and functional effects on protein can contribute to the clinical phenotypes; however, the correlation between genotype and phenotype has not been revealed clearly [4].

With the remarkable advancements in molecular genetic technologies in recent decades, vari- ous genetic testing methods have been developed to elucidate the genetic cause of epilepsy. High-throughput nucleotide sequencing is one of the most useful diagnostic tools because of its cost-effectiveness and high diagnostic yield in patients with early onset epilepsy. This tool has saved time and expense in the diagnosis of genetic causes in genetic epilepsy patients with highly heterogeneous phenotypes [5].

We report a case of SCN2A pathogenic variant in a developmental epileptic encephalopathy patient who was successfully treated with an adequate anti-seizure medication through the epilepsy gene panel testing based on high-throughput nucleotide sequencing method. This study was approved by the Institutional Review Board of Samsung Seoul Hospital (IRB No. 2014-07001-004 and 2019-01-030). Written informed consent by the patients was waived due to a retrospective nature of our study.

A male neonate born at $38^{+1}$ weeks of gestation was referred to another neonatal intensive care unit with seizures that had begun on the day of birth. He was the second baby of the family and was born by vaginal delivery. After birth, he was treated with oxygen with meconium aspiration for several hours. He had no family history

Copyright (C 2021 Korean Child Neurology Society

This is an Open Access article distributed under the terms of the Creative Commons Attribution Non-Commercial License (http://creativecommons.org/licenses/by-nc/4.0/) which permits unrestricted non-commercial use, distribution, and reproduction in any medium, provided the original work is properly cited. 

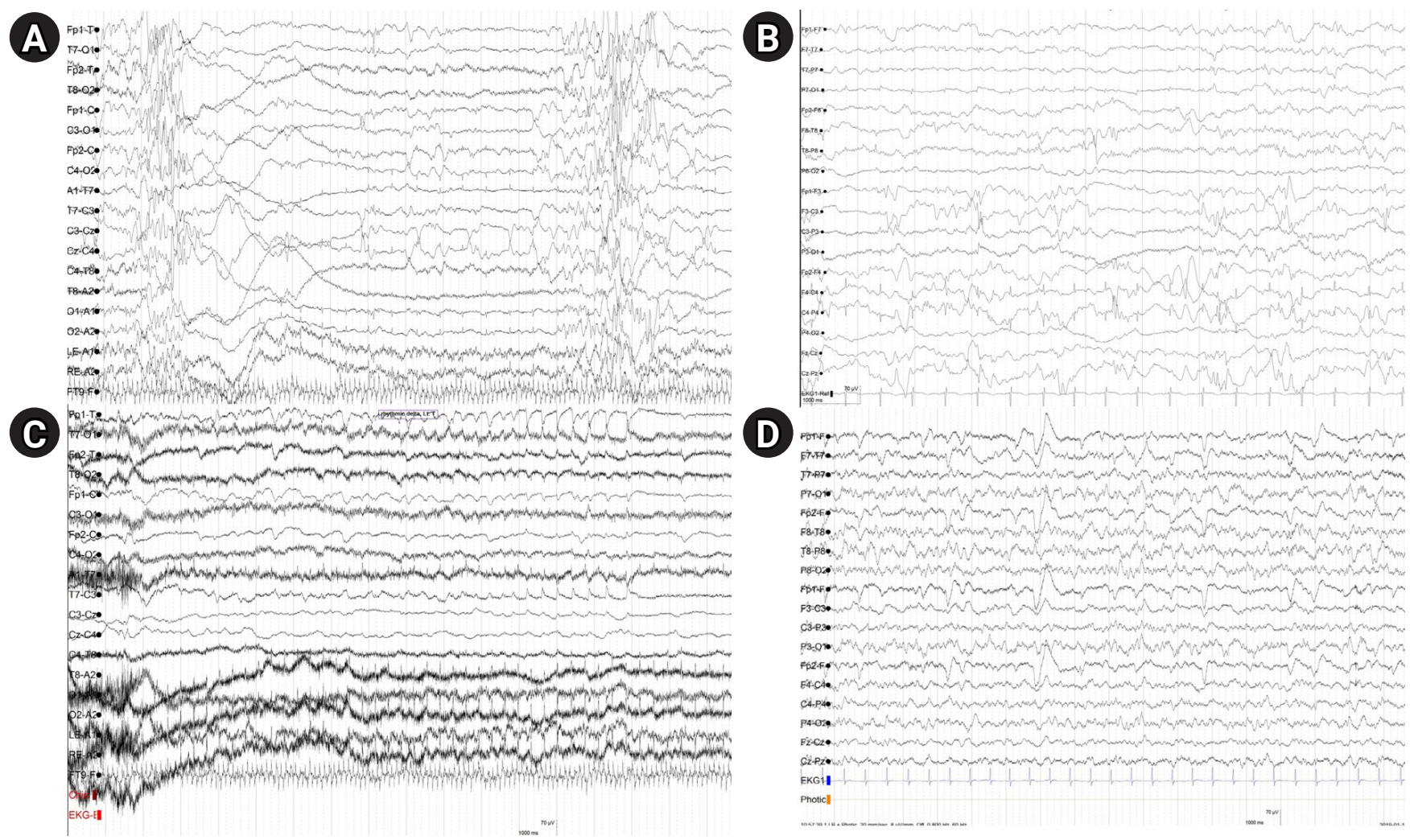

Fig. 1. Electroencephalography of the patient during follow-up. (A, B) The electroencephalography shows generalized spike and wave mixtures, background suppression, spike discharges from the left or right central, or temporal areas. (C) Rhythmic sharp wave discharges from the left central area are seen for about 10 seconds, which is an electroencephalographic seizure. (D) The electroencephalography at 2 years of age is normal.

of neuromuscular, metabolic, or genetic disorders. His weight, height, and head circumference were $2,900 \mathrm{~g}$ (25-75th percentile), $49 \mathrm{~cm}$ (50-75th percentile), and $35 \mathrm{~cm}$ (75-90th percentile), respectively. He showed generalized tonic seizures 15 hours after birth and was evaluated for electrolytes, brain ultrasonography, cerebrospinal fluid analysis, and metabolic abnormalities, all of which were normal. He was then treated with three doses of intravenous phenobarbital (each $10 \mathrm{mg} / \mathrm{kg}$ ). Brain magnetic resonance imaging was normal, and repeated blood tests including electrolytes, ammonia, and metabolic analysis were normal.

He was transferred to this institution on the 4th day after birth. On admission, he showed a mildly hypotonic posture although sucking power was good. There were no anomalies or remarkable findings without caput succedaneum in the vertex area. Neurological examination was normal, and primitive reflexes such as the Moro reflex, sucking reflex, and rooting reflex were present. The initial electroencephalography on arrival revealed multifocal spike or polyspike discharges from the left or right temporal, central, or occipital areas and showed intermittent background suppression lasting about 2 to 5 seconds (Fig. $1 \mathrm{~A}$ and B). There were frequent electro-clinical or electroencephalographic seizures arising from the left temporal areas lasting about 20 to 40 seconds (Fig. 1C). After administration of pyridoxine and levetiracetam $(20 \mathrm{mg} / \mathrm{kg}$, three times), the clinical seizures disappeared. However, electroencephalographic seizures appeared intermittently and were managed with further doses of levetiracetam. On the 7th day in the hospital, the epilepsy gene panel testing based on high-throughput nucleotide sequencing method was performed because there was no remarkable cause found with brain magnetic resonance imaging and metabolic disease screening test. For the intractable seizures, the patient was treated with adding topiramate and a ketogenic diet. After administration of oxcarbazepine on the 30th day in the hospital, the seizures had been controlled clinically and electroencephalographically. However, the electroencephalography showed frequent spike discharges from the left or right temporal or central areas. He was discharged on the 40th day from the hospital after being seizure free for 9 days with multiple anti-seizure medications (phenobarbital, phenytoin, levetiracetam, topiramate, oxcarbazepine, and pyridoxine). After a week, the gene panel testing revealed a pathogenic variant of the SCN2A gene (NM_021007.2: c.4609A > T; p.(Ile537Phe), heterozygous variant), which was found to be de novo after analysis of the parents' DNA. After dis- 


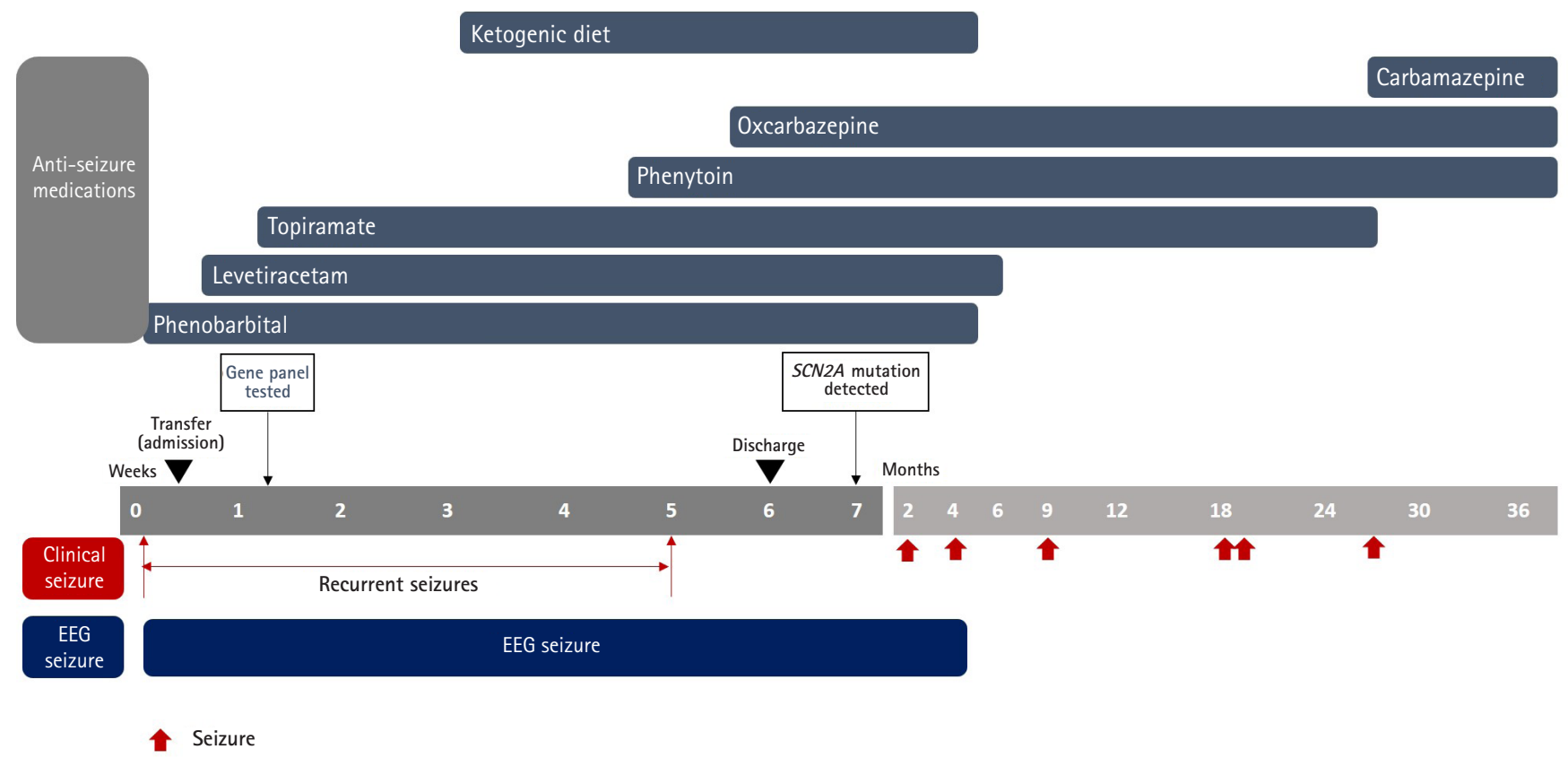

Fig. 2. Summary of clinical and electroencephalographic seizure on administered anti-seizure medications. After finding de novo pathogenic variant of voltage-gated sodium channel, type II, alpha (SCN2A) gene in gene panel testing, sodium channel blockers such as phenytoin, oxcarbazepine, and carbamazepine were administered. Adjusting the dosage of these medications, the patient became seizure free electroencephalographically although there were rare break-through seizures occasionally. EEG, electroencephalography.

charge, the patient showed focal motor seizures lasting about 10 to 70 seconds with a frequency of 2 to 14 times daily. The doses of phenytoin and oxcarbazepine were increased based on the results of the gene panel testing. On the 61st day after birth (19 days after discharge), he became completely seizure free after the phenytoin ( $25 \mathrm{mg}$ three times a day, $14 \mathrm{mg} / \mathrm{kg} /$ day, serum through lev$\mathrm{el}=12.37 \mu \mathrm{g} / \mathrm{mL})$ and oxcarbazepine (120 mg twice a day, 46.2 $\mathrm{mg} / \mathrm{kg} /$ day, serum through level of 10-hydroxy-carbazepine $=8.6$ $\mathrm{mg} / \mathrm{L}$, therapeutic range: 3 to $35 \mathrm{mg} / \mathrm{L}$ ) dosages were adjusted. Occasionally, he showed break-through seizures, which became under control with adjusting the dose of phenytoin. At the age of 27 months, he showed clustering seizures during a febrile illness associated with respiratory syncytial virus infection. At that time, he couldn't take the anti-seizure medication with gastroenteritis associated with viral illness. His seizures were controlled with intravenous administration of phenytoin and his electroencephalography was normal (Fig. 1D). Because the effects of phenytoin and oxcarbazepine were insufficient to control his seizures, we added carbamazepine $(150 \mathrm{mg}$ twice a day, $21.7 \mathrm{mg} / \mathrm{kg} /$ day, serum through level $=2.9 \mu \mathrm{g} / \mathrm{mL}$ ), another sodium channel blocker, after his vomiting and fever subsided. The clinical course was summarized in Fig. 2.

In the last clinical follow-up, he was a 3-year-old and has been seizure free with three anti-seizure medications (carbamazepine, oxcarbazepine, and phenytoin). He showed global developmental delay, could stand with assistance, walk with full assist, express several vowel sounds, and understand several orders such as 'point to your nose'.

In a large cohort study of 201 patients with SCN2A-related disorders, developmental epileptic encephalopathy with early onset before the age of 3 months accounted for $36 \%(n=37)$. Most of them $(\mathrm{n}=31)$ showed their first seizure within a week like our case and their clinical manifestations had a wide range of epilepsy syndromes. The response to sodium channel blockers was very different for each patient. Among sodium channel blockers, phenytoin was the most effective anti-seizure medication. There were eight patients being seizure free with phenytoin and our patient also showed good response to intravenous administration of phenytoin [3].

We report a case of $S C N 2 A$ pathogenic variant treated with an adequate anti-seizure medication based on the findings of highthroughput nucleotide sequencing-based gene panel testing.

\section{Conflicts of interest}

No potential conflict of interest relevant to this article was reported.

\section{ORCID}

Se Eun Park, https://orcid.org/0000-0003-4453-0436 
Jiwon Lee, https://orcid.org/0000-0002-8456-684X

\section{Author contribution}

Conceptualization: JL and JL. Data curation: DL, JYS, and JL. Formal analysis: DL and JYS. Methodology: SEP, DL, and JL. Project administration: JL. Writing-original draft: SEP and JL. Writing-review \& editing: JL and JL.

\section{Acknowledgements}

We appreciate the patient and his family for their participation in this study.

\section{References}

1. Catterall WA. From ionic currents to molecular mechanisms: the structure and function of voltage-gated sodium channels. Neuron 2000;26:13-25.

2. Whitaker WR, Faull RL, Waldvogel HJ, Plumpton CJ, Emson PC, Clare JJ. Comparative distribution of voltage-gated sodium channel proteins in human brain. Brain Res Mol Brain Res 2001;88:37-53.

3. Wolff M, Johannesen KM, Hedrich UBS, Masnada S, Rubboli G, Gardella E, et al. Genetic and phenotypic heterogeneity suggest therapeutic implications in SCN2A-related disorders. Brain 2017;140:1316-36.

4. Reynolds C, King MD, Gorman KM. The phenotypic spectrum of SCN2A-related epilepsy. Eur J Paediatr Neurol 2020; 24:117-22.

5. Lee J, Lee C, Ki CS, Lee J. Determining the best candidates for next-generation sequencing-based gene panel for evaluation of early-onset epilepsy. Mol Genet Genomic Med 2020;8:e1376. 